\title{
Sharp pointwise gradient estimates for Riesz potentials with a bounded density
}

\author{
Vladimir G. Tkachev ${ }^{1}$
}

Received: 26 March 2018 / Revised: 16 April 2018 / Accepted: 20 April 2018 /

Published online: 28 April 2018

(C) The Author(s) 2018

\begin{abstract}
We establish sharp inequalities for the Riesz potential and its gradient in $\mathbb{R}^{n}$ and indicate their usefulness for potential analysis, moment theory and other applications.
\end{abstract}

Keywords Riesz potentials · Exponential transform - $L$-problem of moments · Subharmonic functions · Cauchy's inequality $\cdot$ Symmetry of domains and solutions

Mathematics Subject Classification Primary: 31B15 - 47B06 - 44A60; Secondary: $44 \mathrm{~A} 15$

\section{Introduction}

Given a measurable function $f(x)$ on $\mathbb{R}^{n}$, its Riesz potential of order $0 \leq \alpha<n$ is defined $^{1}$ by

$$
\left(\mathcal{I}_{\alpha} \rho\right)(y)=\int_{\mathbb{R}^{n}} \frac{\rho(x)}{|y-x|^{n-\alpha}} d_{\omega} x,
$$

where $d_{\omega} x$ denotes the $n$-dimensional Lebesgue measure on $\mathbb{R}^{n}$ normalized by

$$
d_{\omega} x=\frac{1}{\omega_{n}} d x,
$$

\footnotetext{
1 The normalization we use is slightly different from the standard normalization [3].

In memory of Sasha Vasil'ev Friend, Colleague, Mathematician.

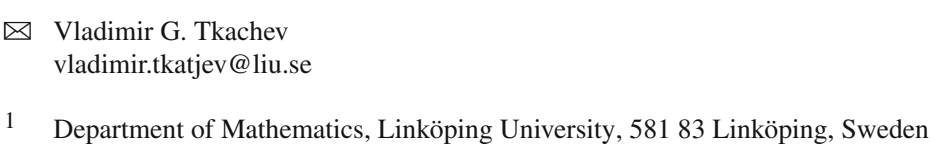


$\omega_{n}:=\pi^{n / 2} / \Gamma\left(\frac{n}{2}+1\right)$ being the $n$-dimensional volume of the unit ball in $\mathbb{R}^{n}$. Let $\rho(x), 0 \leq \rho \leq 1$, be a measurable function with compact support in $\mathbb{R}^{n}$ and let

$$
E_{\rho}(y)=\exp \left[-2 \int_{\mathbb{R}^{n}} \frac{\rho(x)}{|y-x|^{n}} d_{\omega} x\right]=e^{-2\left(\mathcal{I}_{\alpha} \rho\right)(y)}, \quad y \in \mathbb{R}^{n} \backslash \operatorname{supp} \rho,
$$

be the exponential transform of $\rho[9,10]$. Then $E_{\rho}(y)$ is the exponential of a Riesz potential of first nonintegrable index $(\alpha=0)$. If $\rho=\chi_{D}$ is a characteristic function of a bounded domain $D \subset \mathbb{R}^{n}$, Putinar and Gustafsson [9] established that $E_{\chi_{D}}(x)$ is superharmonic in the complement of $D$ and it tends to zero at smooth points of the boundary $\partial D$. It has also been conjectured in [9] that in fact a stronger result holds: for any density $\rho(x)$, the function

$$
\begin{cases}\ln \left(1-E_{\rho}(y)\right), & \text { if } \quad n=2, \\ \frac{1}{n-2}\left(1-E_{\rho}(y)\right)^{(n-2) / n}, & \text { if } \quad n \geq 3\end{cases}
$$

is subharmonic everywhere outside supp $\rho$. The conjecture has been settled in the affirmative by the author in [23]. A key ingredient in the proof of the subharmonicity was the following sharp inequality.

Theorem 1 (Corollary 2.2. in [23]) For any density function $0 \leq \rho(x) \leq 1,0 \notin$ supp $\rho$, the inequality

$$
\left(\int_{\mathbb{R}^{n}} \frac{x_{1} \rho(x)}{|x|^{n}} d_{\omega} x\right)^{2} \leq \mathscr{M}_{n}\left(\int_{\mathbb{R}^{n}} \frac{\rho(x)}{|x|^{n}} d_{\omega} x\right) \int_{\mathbb{R}^{n}} \frac{\rho(x)}{|x|^{n-2}} d_{\omega} x,
$$

holds, where $\mathscr{M}_{n}(t)$ is the unique solution of the initial problem

$$
\mathscr{M}_{n}^{\prime}(t)=1-\mathscr{M}_{n}^{2 / n}(t), \quad \mathscr{M}(0)=0 .
$$

The inequality (2) is sharp and the equality holds when $\rho(x)$ is the characteristic function of ball $B$ with a center on the $x_{1}$-axes and $0 \notin \bar{B}$.

Inequality (2) is remarkable in many respects. First notice that it implies a sharp gradient estimate for the Newtonian potential

$$
\frac{1}{(n-2)^{2}}\left|\nabla \mathcal{I}_{2} \rho(x)\right|^{2} \leq \mathscr{M}\left(\mathcal{I}_{0} \rho(x)\right) \cdot \mathcal{I}_{2} \rho(x),
$$

or

$$
\frac{1}{n-2}\left|\nabla U_{\rho}(y)\right|^{2} \leq U_{\rho}(y) \cdot \mathscr{M}_{n}\left(-\frac{1}{2} \ln E_{\rho}(y)\right), \quad \forall y \notin \operatorname{supp} \rho,
$$

for the Newtonian potential

$$
U_{\rho}(y)=\frac{1}{n-2} \int_{\mathbb{R}^{n}} \frac{\rho(x)}{|y-x|^{n-2}} d_{\omega} x
$$


with a bounded density $\rho$. Since $\mathscr{M}_{n}(t)<1$, (5) yields a 'truncated version'

$$
\left|\nabla U_{\rho}(x)\right|<\sqrt{(n-2) U_{\rho}(x)}, \quad x \notin \operatorname{supp} \rho .
$$

It is well-known, see for example Proposition 3.1.7 in [3], that for $f \in L^{p}\left(\mathbb{R}^{n}\right)$, $1 \leq p<\infty$, there exists a constant $A$ such that

$$
\left|\nabla I_{\alpha} f(x)\right|^{\alpha} \leq A \cdot M f(x) \cdot\left(I_{\alpha}(f)(x)\right)^{\alpha-1},
$$

where

$$
M f(x)=\sup _{r>0} \frac{1}{r^{n} \omega_{n}} \int_{B(x, r)}|f(y)| d y
$$

is the Hardy-Littlewood maximal function of $f$. As Adams remarks in [2], while the maximal function is an important tool for estimates involving $L^{p}$ measures $f$, it is not a sharp tool for analyzing their pointwise behaviour. Some generalizations involving the Hardy-Littlewood maximal function for a complex order $\alpha \in \mathbb{C}$ can be found in [19]. Estimate (6) also appears in the Poisson equation and quasilinear equations, see [20,21], see also [7]. In fact, a straightforward application of Cauchy's inequality yields just

$$
\left(\int_{\mathbb{R}^{n}} \frac{x_{1} \rho(x)}{|x|^{n}} d_{\omega} x\right)^{2} \leq \int_{\mathbb{R}^{n}} \frac{\rho(x)}{|x|^{n}} d_{\omega} x \int_{\mathbb{R}^{n}} \frac{\rho(x)}{|x|^{n-2}} d_{\omega} x
$$

which is optimal in the class of arbitrary (not uniformly bounded) nonnegative measurable densities $\rho$ and the equality is attained asymptotically for a suitable approximation of a $\delta$-function distribution with a single-point support on the $x_{1}$-axes.

In this respect, (2) is a considerable refinement of (7) for uniformly bounded densities $0 \leq \rho \in L^{\infty}\left(\mathbb{R}^{n}\right)$. Since $\mathscr{M}_{n}<1$ one readily obtains from (2) that for any $0 \leq \rho \in L^{\infty}\left(\mathbb{R}^{n}\right)$ the (truncated) Cauchy inequality holds:

$$
\left(\int_{\mathbb{R}^{n}} \frac{x_{1} \rho(x)}{|x|^{n}} d_{\omega} x\right)^{2}<\|\rho\|_{L^{\infty}\left(\mathbb{R}^{n}\right)} \int_{\mathbb{R}^{n}} \frac{\rho(x)}{|x|^{n-2}} d_{\omega} x,
$$

Observe that the sharp inequality (2) has no longer the symmetry of Cauchy's inequality, see (7). This symmetry breaking can appropriately be explained in the moment problem context. Namely, (2) can be thought as a natural extension of the Markov inequalities in the $L$-problem $[4,15]$ for the critical negative powers. Recall that given $L>0$, the $L$-problem of moments concerns the existence of a density function $0 \leq \rho \leq L$ with a given sequence of the power moments

$$
s_{k}(\rho):=s_{k}=\int_{I} \rho(x) x^{k} d x, \quad k=0,1,2, \ldots,
$$

where $I \subset \mathbb{R}$ is an arbitrary fixed (finite or infinite) interval. By a celebrated result of A.A. Markov, the solvability of the $L$-problem is equivalent to the solvability of the corresponding classical moment problem for 


$$
a_{k}=\int_{I} x^{k} d \mu(x)
$$

where $d \mu(x)$ is a positive measure, and the correspondence is given by the (onedimensional) exponential transform

$$
1-\exp \left(-\frac{1}{L} \sum_{k=0}^{\infty} \frac{s_{k}}{z^{k+1}}\right)=\sum_{k=0}^{\infty} \frac{a_{k}}{z^{k+1}}
$$

see [4, p. 72], [15, p. 243], [8] for more details. Setting $L=1$ and $I=[0, \infty)$ in (9), the solvability of the corersponding $L$-moment problem is equivalent to the solvability of the Stieltjes problem for the sequence $\left\{a_{k}\right\}_{k \geq 0}$ defined by (10) which is, in its turn, is equivalent to the nonnegativity of the Hankel determinants sequence $\Delta_{m}:=\operatorname{det}\left(a_{i+j}\right)_{i, j=0}^{m} \geq 0$ and $\Delta_{m}^{\prime}:=\operatorname{det}\left(a_{i+j+1}\right)_{i, j=0}^{m} \geq 0, m \geq 0$. For example, $\Delta_{1}^{\prime} \geq 0$ readily yields

$$
s_{0}^{2} \leq 12\left(s_{0} s_{2}-s_{1}^{2}\right)
$$

Furthermore, the inversion of the variable $x \rightarrow 1 / x$ in (9) implies a correspondence between the negative power moments $s_{m}$ for $m=-2,-3, \ldots$ and the classical ones:

$$
s_{k}(\rho)=s_{-k-2}(\widetilde{\rho}), \quad \tilde{\rho}(t)=\rho\left(x^{-1}\right) .
$$

This implies the classical Markov inequalities for all power moments $s_{m}$ except for the critical exponent $m=-1$.

In this respect, in the one-dimensional case (2) provides a novel inequality for power $L$-moments involving the critical exponent $s_{-1}$. Indeed, in the notation of Theorem 1 one has $\mathscr{M}_{1}(t)=\tanh t$ and $d_{\omega} x=\frac{1}{2} d x$, hence (2) yields

$$
\frac{1}{2}\left(\int_{0}^{\infty} \rho(x) d x\right)^{2} \leq \tanh \left(\frac{1}{2} \int_{0}^{\infty} \frac{\rho(x)}{x} d x\right) \cdot \int_{0}^{\infty} x \rho(x) d x
$$

for any density function $0 \leq \rho \leq 1$. In the moment notation (12) this yields a sharp inequality

$$
s_{0}^{2} \leq s_{1} \tanh s_{-1}
$$

Remark that in contrast to the algebraic character of the classical Markov inequalities [5], (14) has a different, transcentental nature. The analogous two-dimensional $L$ problem is much less explored, recent works point out some direct applications of this problem to tomography, geophysics, the problem in particular has to do with the distribution of pairs of random variables or the logarithmic potential of a planar domain, see [10-12,16]. 


\section{Main results}

In this paper we extend (2) to the Riesz potentials of a general index. Then we have for its gradient

$$
\frac{1}{n-\alpha}\left(\nabla \mathcal{I}_{\alpha} \rho\right)(y)=\int_{\mathbb{R}^{n}} \frac{(y-x) \rho(x)}{|y-x|^{n+2-\alpha}} d_{\omega} x .
$$

When $y$ is fixed it is natural to assume that $y=0$, hence after a suitable orthogonal transformation of $\mathbb{R}^{n}$ the above integrals amount respectively to

$$
\begin{aligned}
\mathcal{F}_{\alpha} \rho & :=\int_{\mathbb{R}^{n}} \frac{\rho(x)}{|x|^{n-\alpha}} d_{\omega} x, \\
\mathcal{H}_{\alpha} \rho & :=\int_{\mathbb{R}^{n}} \frac{x_{1} \rho(x)}{|x|^{n+2-\alpha}} d_{\omega} x,
\end{aligned}
$$

with a new density function $\rho$. We are interested in the gradient estimates, i.e. those involving both $\mathcal{F}_{\alpha} \rho$ and $\mathcal{H}_{\alpha} \rho$. In this paper, we consider the following variational problem.

Definition 1 Given $u, v>0$, define

$$
\mathscr{N}_{\alpha}(u, v):=\sup _{\rho}\left\{\left|\mathcal{H}_{\alpha} \rho\right|^{2}: \mathcal{F}_{\alpha} \rho=u, \quad \mathcal{F}_{\alpha-2} \rho=v\right\}
$$

where the supremum is taken over all measurable functions $0 \leq \rho \leq 1$ with support outside of the origin. We refer to such a $\rho(x)$ as an admissible density function. A pair $(u, v) \subset \mathbb{R}_{>0}^{2}$ is said to be admissible for the variational problem (16) if there exists an admissible density function $\rho$ such that $\mathcal{F}_{\alpha} \rho=u$ and $\mathcal{F}_{\alpha-2} \rho=v$.

It is easy to see that $\mathscr{N}_{\alpha}(u, v)$ is well-defined and finite for any $\alpha$ and any admissible pair $(u, v)$. Indeed, it follows from Cauchy's inequality that

$$
\mathscr{N}_{\alpha}(u, v) \leq u v, \quad \forall \alpha \in \mathbb{R}
$$

We point out, however, that the estimate (17) provide a correct approximation only when $u$ and $v$ are infinitesimally small.

Combining (15) with (16) yields the following pointwise gradient estimate on the Riesz potential $\mathcal{I}_{\alpha} \rho$ by means of $\mathcal{I}_{\alpha} \rho$ itself and the contiguous potential $\mathcal{I}_{\alpha-2} \rho$.

Proposition 1 In the above notation, the following pointwise estimate holds:

$$
\frac{1}{n-\alpha}\left|\nabla \mathcal{I}_{\alpha} \rho\right| \leq \sqrt{\mathscr{N}_{\alpha}\left(\mathcal{I}_{\alpha} \rho, \mathcal{I}_{\alpha-2} \rho\right)}
$$

and the inequality is sharp.

Our main result provides an explicit form of the goal function $\mathscr{N}_{\alpha}(u, v)$. 
Theorem 2 Let $n \geq 1$ and $0<\alpha \leq 2$. Then the set of admissible pairs coincides with the nonnegative quadrant $\mathbb{R}_{\geq 0}^{2}$ and for any $u, v>0$

$$
\mathscr{N}_{\alpha}(u, v)=u^{2(\alpha-1) / \alpha} \frac{h_{\alpha}^{2}(t)}{f_{\alpha}^{2(\alpha-1) / \alpha}(t)},
$$

where $t=t(u, v)$ is uniquely determined by the relation

$$
f_{\alpha}^{2-\alpha}(t) f_{\alpha-2}^{\alpha}(t)=u^{2-\alpha} v^{\alpha}
$$

where

$$
\begin{aligned}
& f_{\alpha}(t)=t^{2-n}\left(t^{2}-1\right)^{n / 2} F\left(\frac{2-\alpha}{2}, \frac{2+\alpha}{2} ; \frac{n+2}{2}, 1-t^{2}\right) \\
& h_{\alpha}(t)=t^{1-n}\left(t^{2}-1\right)^{n / 2} F\left(\frac{2-\alpha}{2}, \frac{\alpha}{2} ; \frac{n+2}{2}, 1-t^{2}\right),
\end{aligned}
$$

and $F([a, b],[c], t)$ is the Gauss hypergeometric function.

We are in particular interested in the shape structute of the goal function $\mathscr{N}_{\alpha}(u, v)$, i.e. how it depends on the variables $u$ and $v$. Combining (20) and (19) yields the following alternative representation.

Corollary 1 Under assumptions of Theorem 2,

$$
\mathscr{N}_{\alpha}(u, v)=u v \cdot \psi\left(u^{2-\alpha} v^{\alpha}\right),
$$

where $\psi(s)$ well-defined by the parametric representation

$$
\psi(s)=\frac{h_{\alpha}^{2}(t)}{f_{\alpha}(t) f_{\alpha-2}(t)}, \quad s=f_{\alpha}^{2-\alpha}(t) f_{\alpha-2}^{\alpha}(t) .
$$

Some further remarks are in order. The condition $\alpha \leq 2$ in Theorem 2 is of a technical character. In the complementary case $2<\alpha<n$, the set of admissible pairs is a proper subset of the quadrant $\mathbb{R}_{\geq 0}^{2}$. The corresponding analysis requires some more care, and will be done elsewhere.

The borderline case $\alpha=2$ corresponds to inequality (2) established in [23] and in the present notation the goal function here becomes

$$
\mathscr{N}_{2}(u, v)=u \cdot \mathscr{M}_{n}(v) .
$$

We derive also it as an application of Theorem 2 in Corollary 2 below. Remarkably, that both Cauchy's inequality estimate (17) and its sharp version (22) separate into one-variable factors. This separable form, however, no longer holds for a general $\alpha$, when the shape of $\mathscr{N}_{\alpha}$ has a more complicated structure, see (21). 
Another interesting case is $\alpha=1$. Under this condition, the Riesz potentials in the right hand side of (18) have in fact the same exponent because for $\alpha=1$ the contiguous potential amounts to $\mathcal{I}_{-1} \rho=\mathcal{I}_{1} \widetilde{\rho}$, where $\widetilde{\rho}$ is the inversion of $\rho$. A further remarkable feature of this case is that $\mathscr{N}_{1}(u, v)$ becomes a symmetric function of $u$ and $v$. Indeed, a straightforward analysis of (21) implies that for $\alpha=1$ the goal function $\mathscr{N}_{1}(u, v)$ depends only on the product $u v$. More precisely, we have the following

Theorem 3 For any measurable function $0 \leq \rho(x) \leq 1$, $0 \notin$ supp $\rho$, the sharp inequality holds

$$
\left|\int_{\mathbb{R}^{n}} \frac{x_{1} \rho(x)}{|x|^{n+1}} d_{\omega} x\right| \leq \Phi_{n}\left(\sqrt{\int_{\mathbb{R}^{n}} \frac{\rho(x)}{|x|^{n-1}} d_{\omega} x \cdot \int_{\mathbb{R}^{n}} \frac{\rho(x)}{|x|^{n+1}} d_{\omega} x}\right),
$$

where $\Phi_{n}(t)$ is the unique solution of the initial problem

$$
\Phi_{n}^{\prime \prime}=\frac{\Phi_{n}^{\prime}\left(\Phi_{n}^{\prime 2}-1\right)}{(n-1) \Phi_{n} \Phi_{n}^{\prime}+s}, \quad \Phi_{n}(0)=0, \quad \Phi_{n}^{\prime}(0)=1
$$

subject to the asymptotic condition

$$
\lim _{s \rightarrow \infty} \frac{\Phi_{n}(s)}{\ln s}=\frac{\Gamma\left(\frac{n+2}{2}\right)}{\Gamma\left(\frac{n+1}{2}\right) \Gamma\left(\frac{3}{2}\right)} .
$$

Remark 1 Concerning the definition of the shape function $\Phi_{n}$, we note that the initial problem (24) itself does not determine a unique solution because the initial condition $\Phi_{n}^{\prime}(0)=1$ is singular. One can prove that if $\phi(x)$ solves (24) then any solution of (24) is obtained by the homothetic scaling $\frac{1}{c} \phi(c x), c>0$. Note also that all thus obtained solutions have the logarithmic growth at infinity, so a further normalization like (25) is needed.

The proof of Theorem 2 relies on a refinement of the technique initiated in [23] and uses the Bathtub principle. We obtain some preliminary results in Sect. 3 and finish the proof in Sect. 5. Then we prove Theorem 3 in Sect. 7.

Remark also that the obtained gradient estimates are sharp for Euclidean balls with constant density. The latter symmetry phenomenon is natural for the Riesz and Newton potentials $[6,18]$, and studied recently in connection with Riesz potential integral equations on exterior domains [13, 14,22,24].

We finally mention that our results can also be thought of as an analogue of the polynomial moment inequalities for the singular Riesz potential $d \mu_{\alpha}(x)=|x|^{\alpha-n}$ in $\mathbb{R}^{n}$. Then the above functionals are recognized as the lower order polynomial moments:

$\mathcal{F}_{\alpha} \rho=\int 1 d \mu_{\alpha}(x), \quad \mathcal{H}_{\alpha} \rho=\int x_{1} d \mu_{\alpha}(x), \quad \mathcal{F}_{\alpha-2} \rho=\int\left(x_{1}^{2}+\ldots+x_{n}^{2}\right) d \mu_{\alpha}(x)$

It is natural to speculate what is the natural extension of the Hankel determinant inequalities for $d \mu_{\alpha}$. We pursue this theme elsewhere. 


\section{Auxiliary identities for spherical integrals}

Let us decompose $\mathbb{R}^{n}=\mathbb{R} \times \mathbb{R}^{n-1}$ such that $x=\left(x_{1}, y\right)$, where $y=\left(x_{2}, \ldots, x_{n}\right) \in$ $\mathbb{R}^{n-1}$. Given $0<\sigma<\tau$, let $B(\tau, \sigma)$ denote the open ball of radius $\sqrt{\tau^{2}-\sigma^{2}}$ centered at $(\tau, 0) \in \mathbb{R}^{n}$, i.e.

$$
B(\tau, \sigma)=\left\{x=\left(x_{1}, y\right) \in \mathbb{R}^{n}:|x|^{2}-2 \tau x_{1}+\sigma^{2}<0\right\},
$$

and let $D(t):=B(t, 1)$. We refer to $B(\tau, \sigma)$ as an $x_{1}$-ball. It is easy to see and will be used later that the inversion $x \rightarrow x^{*}=x /|x|^{2}$ acts on $x_{1}$-balls as follows:

$$
B(\tau, \sigma)^{*}=B\left(\frac{\tau}{\sigma^{2}}, \frac{1}{\sigma}\right), \quad D(t)^{*}=D(t) .
$$

Let us fix some notation:

$$
\begin{aligned}
& F_{\alpha}(\tau, \sigma) \equiv \mathcal{F}_{\alpha} \chi_{B(\tau, \sigma)}=\int_{B(\tau, \sigma)} \frac{1}{|x|^{n-\alpha}} d_{\omega} x=\sigma^{\alpha} f_{\alpha}\left(\frac{\tau}{\sigma}\right) \\
& H_{\alpha}(\tau, \sigma) \equiv \mathcal{H}_{\alpha} \chi_{B(\tau, \sigma)}=\int_{B(\tau, \sigma)} \frac{x_{1}}{|x|^{n+2-\alpha}} d_{\omega} x=\sigma^{\alpha-1} h_{\alpha}\left(\frac{\tau}{\sigma}\right)
\end{aligned}
$$

where

$$
f_{\alpha}(t):=F_{\alpha}(t, 1), \quad h_{\alpha}(t):=H_{\alpha}(t, 1) .
$$

All the introduced functions depend also on the ambient dimension $n$.

First notice that $f_{\alpha}(t)$ and $h_{\alpha}(\alpha)$ are real analytic functions of $t>0$, and for any real $\alpha$

$$
\lim _{t \rightarrow \infty} f_{\alpha}(t)=\int_{x_{1}>0} \frac{d_{\omega} x}{|x|^{n-\alpha}}=\infty .
$$

Applying Stokes' formula to

$$
0=\int_{B(\tau, \sigma)} \operatorname{div}\left(\frac{|x|^{2}-2 \tau x_{1}+\sigma^{2}}{|x|^{n-\alpha+2}} \cdot x\right) d_{\omega} x
$$

we get the following identity:

$$
\alpha F_{\alpha}(\tau, \sigma)+(\alpha-2) \sigma^{2} F_{\alpha-2}(\tau, \sigma)=2(\alpha-1) \tau H_{\alpha}(\tau, \sigma)
$$

Further, applying the inversion readily yields by virtue of (26) that

$$
F_{\alpha}(\tau, \sigma)=F_{-\alpha}\left(\frac{\tau}{\sigma^{2}}, \frac{1}{\sigma}\right), \quad H_{\alpha}(\tau, \sigma)=H_{2-\alpha}\left(\frac{\tau}{\sigma^{2}}, \frac{1}{\sigma}\right) .
$$


For the reduced functions this amounts to

$$
\begin{aligned}
2(\alpha-1) t h_{\alpha}(t) & =\alpha f_{\alpha}(t)+(\alpha-2) f_{\alpha-2}(t), \\
f_{\alpha}(t) & =f_{-\alpha}(t), \\
h_{\alpha}(t) & =h_{2-\alpha}(t)
\end{aligned}
$$

Lemma 1 For any $\alpha \in \mathbb{R}$, the following identities hold:

$$
\begin{aligned}
& 2 t h_{\alpha}^{\prime}=f_{\alpha}^{\prime}+f_{\alpha-2}^{\prime}, \\
& (n-\alpha) h_{\alpha}=\left(t^{2}-1\right) f_{\alpha}^{\prime}-\alpha t f_{\alpha}, \\
& \lim _{t \rightarrow 1+0} f_{\alpha}(t)\left(1-t^{2}\right)^{-n / 2}=1 .
\end{aligned}
$$

Proof Let us consider an axillary function $\lambda(x)=\left(|x|^{2}+1\right) / 2 x_{1}$. Then $x_{1} \nabla \lambda+\lambda e_{1}=$ $x$, hence

$$
\nabla \lambda=\frac{x-\lambda e_{1}}{x_{1}}
$$

and

$$
x_{1}^{2}|\nabla \lambda|^{2}=|x|^{2}-2 \lambda x_{1}+\lambda^{2}=\lambda^{2}-1
$$

Notice that the $s$-level set $\left\{x \in \mathbb{R}^{n}: \lambda(x)=s\right\}$ is exactly the boundary sphere $\partial D(s)$, therefore $\lambda$ foliates the punctured ball $D(t) \backslash\{(1,0)\}$ into the family of spheres $\{\partial D(s): 1<s \leq t\}$. Applying the co-area formula one obtains from (27) and (36)

$$
f_{\alpha}(t)=\frac{1}{\omega_{n}} \int_{1}^{t} d s \int_{\partial D(s)} \frac{d A}{|x|^{n-\alpha}|\nabla \lambda(x)|}=\int_{1}^{t} \frac{d s}{\sqrt{s^{2}-1}} \int_{\partial D(s)} \frac{x_{1}}{|x|^{n-\alpha}} d_{\omega} A
$$

where $d A$ is the $(n-1)$-dimensional surface measure on $\partial D(s)$ and $d_{\omega} A=\frac{1}{\omega_{n}} d A$. In particular,

$$
f_{\alpha}^{\prime}(t)=\frac{1}{\sqrt{t^{2}-1}} \int_{\partial D(t)} \frac{x_{1}}{|x|^{n-\alpha}} d_{\omega} A
$$

Similarly one finds

$$
\begin{aligned}
h_{\alpha}(t) & =\int_{1}^{t} \frac{d t}{\sqrt{t^{2}-1}} \int_{\partial D(s)} \frac{x_{1}^{2}}{|x|^{n+2-\alpha}} d_{\omega} A \\
& =\int_{1}^{t} \frac{d t}{2 t \sqrt{t^{2}-1}} \int_{\partial D(s)} \frac{x_{1}\left(|x|^{2}+1\right)}{|x|^{n+2-\alpha}} d_{\omega} A .
\end{aligned}
$$

Differentiating the obtained identity and applying (37) yields (32).

Next, $\left.\lambda\right|_{\partial D(s)}=s$, therefore the outward normal vector $v$ along the boundary $\partial D(s)$ is found from (35) by

$$
v=\frac{\nabla \lambda}{|\nabla \lambda|}=\frac{x-s e_{1}}{\sqrt{s^{2}-1}}
$$


thus, using identities

$$
\operatorname{div} \frac{x}{|x|^{n-\alpha}}=\frac{\alpha}{|x|^{n-\alpha}}, \quad \operatorname{div} \frac{e_{1}}{|x|^{n-\alpha}}=-\frac{(n-\alpha) x_{1}}{|x|^{n+2-\alpha}}
$$

and applying Stoke's formula we obtain by virtue of (37)

$$
\begin{aligned}
\alpha t f_{\alpha}(t)+(n-\alpha) h_{p+2}(t) & =\int_{D(t)}\left(\frac{\alpha t}{|x|^{n-\alpha}}-\frac{(n-\alpha) x_{1}}{|x|^{n+2-\alpha}}\right) d_{\omega} x \\
& =\int_{\partial D(t)} \frac{\left\langle t x-e_{1} ; \nu\right\rangle}{|x|^{n-\alpha}} d_{\omega} A \\
& =\sqrt{t^{2}-1} \int_{\partial D(t)} \frac{x_{1} d_{\omega} A}{|x|^{n-\alpha}} \\
& =\left(t^{2}-1\right) f_{\alpha}^{\prime}(t)
\end{aligned}
$$

which proves (33). Finally, it follows from (37) that

$$
\begin{aligned}
\lim _{t \rightarrow 1+0} f_{\alpha}^{\prime}(t)\left(t^{2}-1\right)^{(2-n) / 2} & =\lim _{t \rightarrow 1+0} \frac{1}{\left(t^{2}-1\right)^{(n-1) / 2}} \int_{\partial D(t)} \frac{x_{1}}{|x|^{n-\alpha}} d_{\omega} A \\
& =\frac{1}{\omega_{n}} \lim _{t \rightarrow 1+0}|\partial D(t)| \cdot\left(t^{2}-1\right)^{(1-n) / 2}=n
\end{aligned}
$$

Then (34) follows from $f_{\alpha}(1)=0$ and the previous identity by virtue of l'Hospital's rule.

Lemma 2 If $0<\gamma<1$ then for any $p$, $q$ there holds that

$$
f_{p}^{\gamma}(t) f_{q}^{1-\gamma}(t) \geq f_{\gamma p+(1-\gamma) q}(t)
$$

Proof A straightforward corollary of (27) and the Hölder inequality.

\section{The reduced functions via hypergeometric functions}

Differentiating the first identity in (31) followed by elimination of $f_{\alpha-2}, f_{\alpha-2}^{\prime}$ and $h_{\alpha}^{\prime}$ by virtue of (32) and (33) readily yields the following identity:

$$
t\left(t^{2}-1\right) f_{\alpha}^{\prime \prime}+\left(t^{2}-n+1\right) f_{\alpha}^{\prime}-t \alpha^{2} f_{\alpha}=0 .
$$

Setting

$$
f_{\alpha}(t)=\left(t^{2}-1\right)^{n / 2} \phi_{\alpha}\left(t^{2}\right)
$$

the equation (39) is transformed to the hypergeometric differential equation

$$
z(1-z) \phi_{\alpha}^{\prime \prime}(z)+\left(\frac{n}{2}-(1+n) z\right) \phi_{\alpha}^{\prime}(z)-\frac{(n+\alpha)(n-\alpha)}{4} \phi_{\alpha}(z)=0 .
$$


with

$$
a=\frac{n-\alpha}{2}, \quad b=\frac{n+\alpha}{2}, \quad c=\frac{n}{2} .
$$

By (34), $\phi_{\alpha}(1)=1$. Since $\phi_{\alpha}(z)$ is regular at $z=1$, it follows from a Kummer transformation (see formula 15.5 .5 in $[1$, p. 563]) that

$$
\phi_{\alpha}(z)=F\left(\frac{1}{2}(n-\alpha), \frac{1}{2}(n+\alpha) ; \frac{1}{2} n+1,1-z\right), \quad z \geq 1 .
$$

Using another Kummer transformation (formula 15.5.11 in [1, p. 563]) yields an alternative representation

$$
\phi_{\alpha}(z)=z^{-\frac{n+\alpha}{2}} F\left(\frac{n+\alpha}{2}, \frac{2+\alpha}{2} ; \frac{n+2}{2}, \frac{z-1}{z}\right), \quad z \geq 1 .
$$

The latter representation is useful for the asymptotic behavior of $f_{\alpha}$ at $\infty$. From (42) we obtain

$$
f_{\alpha}(t)=t^{-n-\alpha}\left(t^{2}-1\right)^{n / 2} F\left(\frac{n+\alpha}{2}, \frac{2+\alpha}{2} ; \frac{n+2}{2}, \frac{t^{2}-1}{t^{2}}\right) .
$$

In particular, using the Gauss type identity

$$
\lim _{z \rightarrow 1}(1-z)^{a+b-c} F(a, b ; c ; z)=\frac{\Gamma(c) \Gamma(a+b-c)}{\Gamma(a) \Gamma(b)}, \quad c<a+b
$$

we have from (43) the following asymptotic growth:

$$
\lim _{t \rightarrow \infty} \frac{f_{\alpha}(t)}{t^{\alpha}}=\frac{\Gamma\left(\frac{1}{2} n+1\right) \Gamma(\alpha)}{\Gamma\left(\frac{1}{2}(n+\alpha)\right) \Gamma\left(\frac{1}{2} \alpha+1\right)} .
$$

Also, applying a linear transformation (formula 15.3.5 in [1, p. 559]) to (43) yields

$$
f_{\alpha}(t)=t^{2-n}\left(t^{2}-1\right)^{n / 2} F\left(\frac{2-\alpha}{2}, \frac{2+\alpha}{2} ; \frac{n+2}{2}, 1-t^{2}\right) .
$$

A similar argument also works for $h_{\alpha}$ : eliminating $f_{\alpha-2}$ from (32) by virtue of (31) 1 yields

$$
f_{\alpha}(t)=\frac{t^{2}-1}{\alpha} h_{\alpha}^{\prime}(t)+\frac{1}{\alpha t}\left((\alpha-1) t^{2}+1-n\right) h_{\alpha}(t),
$$

therefore (32) amounts to

$$
t^{2}\left(t^{2}-1\right) h_{\alpha}^{\prime \prime}(t)+t\left(t^{2}+1-n\right) h_{\alpha}^{\prime}(t)+\left(n-1-(\alpha-1)^{2} t^{2}\right) h_{\alpha}(t)=0 .
$$


The a substitution $h_{\alpha}(t)=t\left(t^{2}-1\right)^{n / 2} \psi_{\alpha}\left(t^{2}\right)$ transforms the latter equation into a hypergeometric one:

$$
z(1-z) \psi_{\alpha}^{\prime \prime}(z)+\left(1+\frac{n}{2}-(n+2) z\right) \psi_{\alpha}^{\prime}(z)-\frac{(n+\alpha)(n+2-\alpha)}{4} \psi_{\alpha}(z)=0
$$

with

$$
a^{\prime}=\frac{n+2-\alpha}{2}, \quad b^{\prime}=\frac{n+\alpha}{2}, \quad c^{\prime}=\frac{n+2}{2} .
$$

For the same reasons as above, we obtain

$$
\psi_{\alpha}(z)=F\left(\frac{1}{2}(n+2-\alpha), \frac{1}{2}(n+\alpha) ; \frac{1}{2} n+1,1-z\right), \quad z \geq 1,
$$

and

$$
h_{\alpha}(t)=t^{1-n}\left(t^{2}-1\right)^{n / 2} F\left(\frac{2-\alpha}{2}, \frac{\alpha}{2} ; \frac{n+2}{2}, 1-t^{2}\right) .
$$

Let us consider some particular cases when $f_{\alpha}$ can be explicitly specified. When $n=1$ and $\alpha$ is arbitrary, one easily finds from (27) that

$$
f_{\alpha}(t)= \begin{cases}\frac{1}{\alpha} \sinh \alpha \xi, & \text { if } \alpha \neq 0 \\ \xi, & \text { if } \alpha=0\end{cases}
$$

and $h_{\alpha}(t)=f_{\alpha-1}(t)$, where

$$
t=\cosh \xi, \quad 0 \leq \xi<\infty
$$

This yields

$$
f_{\alpha}(t)=\frac{\left(t+\sqrt{t^{2}-1}\right)^{\alpha}-\left(t-\sqrt{t^{2}-1}\right)^{\alpha}}{2 \alpha}
$$

When $\alpha=2$, the spherical mean property for harmonic functions $|x|^{2-n}$ and $x_{1}|x|^{-n}$ was used in [23, Sec. 2.2] to obtain explicit expressions

$$
f_{2}(t)=\left(t^{2}-1\right)^{n / 2} t^{2-n}, \quad h_{2}(t)=\frac{1}{t} f_{2}(t)
$$

and

$$
f_{0}(t)=\int_{1}^{t}\left(s^{2}-1\right)^{(n-2) / 2} s^{1-n} d s .
$$

Another interesting particular case is $\alpha=1$, we have by (31) 1 that $f_{-1}=f_{1}$, and the reduced functions $f_{1}$ and $h_{1}$ can be determined explicitly at least when $n$ is an odd integer. 


\section{Proof of Theorem 2}

Step 1. First we assume that supp $\rho(x) \subset \mathbb{R}_{x_{1}>0}^{n}$ and let $\mathscr{N}_{\alpha}^{+}(u, v)$ denote the corresponding supremum in (16). We claim that for any $u, v>0$ there exist $0<\sigma<\tau$ such that

$$
F_{\alpha}(\tau, \sigma)=u, \quad F_{\alpha-2}(\tau, \sigma)=v,
$$

in other words, the pair $(u, v)$ is admissible by a characteristic function of an $x_{1}$ centered ball. Indeed, rewrite (55) by virtue of the reduced functions as the system

$$
\left\{\begin{array}{l}
f_{\alpha}(t)=u \sigma^{-\alpha} \\
f_{\alpha-2}(t)=v \sigma^{2-\alpha}
\end{array}\right.
$$

where $t=\frac{\tau}{\sigma}$. Consider an auxiliary function

$$
g(t)=f_{\alpha}^{2-\alpha}(t) f_{\alpha-2}^{\alpha}(t)
$$

It follows from $0<\alpha<2$ that $g(t)$ is an increasing function of $t$ and by virtue (34) we have $\lim _{t \rightarrow 1+0} g(t)=0$. Furthermore, setting $\gamma=\frac{2-\alpha}{2}, p=\alpha$ and $q=\alpha-2$ in (38) yields under the made assumptions that

$$
g(t)=f_{\alpha}^{2-\alpha}(t) f_{\alpha-2}^{\alpha}(t) \geq f_{0}^{2}(t),
$$

hence (28) implies that $\lim _{t \rightarrow \infty} g(t)=\infty$. Thus, $g$ is a bijection of $[1, \infty)$ onto $[0, \infty)$.

Now, let $t=t_{0}$ be the unique solution of $g(t)=u^{2-\alpha} v^{\alpha}$ and let

$$
\sigma_{0}:=f_{\alpha}^{-\frac{1}{\alpha}}\left(t_{0}\right) u^{\frac{1}{\alpha}}
$$

Then it follows from (56) and (30) that $\sigma_{0}$ and $\tau_{0}=\sigma_{0} t_{0}$ is a (unique) solution of (55). This proves our claim, and also implies that the set of admissible pairs $(u, v)$ coincides with the nonnegative quadrant $\mathbb{R}_{\geq 0}^{2}$.

In the introduced notation, let $\rho_{0}(x)=\bar{\chi}_{B\left(\tau_{0}, \sigma_{0}\right)}(x)$. Then $\rho_{0}(x)$ is a test function for (16). Thus, using (27) we find

$$
\mathscr{N}_{\alpha}^{+}(u, v) \geq\left(\mathcal{H}_{\alpha} \rho_{0}\right)^{2}=\sigma_{0}^{2(\alpha-1)} h_{2}^{\alpha}\left(t_{0}\right) .
$$

On the other hand, if $\rho(x)$ is an arbitrary test function for (16) then by our choice, both $\rho$ and $\rho_{0}$ are test functions in the auxiliary problem

$$
A=\sup \left\{\int \rho(x) \phi(x) d \mu(x): \int \rho(x) d \mu(x)=\sigma_{0}^{2} u+v, \quad 0 \leq \rho \leq 1\right\}
$$

where $\phi(x)=\frac{x_{1}}{|x|^{2}+\sigma_{0}^{2}}$ and $d \mu(x)=\left(\frac{\sigma_{0}^{2}}{|x|^{n-\alpha}}+\frac{1}{|x|^{n+2-\alpha}}\right) d_{\omega} x$. In particular, we have

$$
\mathscr{N}_{\alpha}^{+}(u, v) \leq A^{2}
$$


By the 'Bathtub principle' [17, p. 28], a solution of the variational problem (59) is given by the characteristic function of a sublevel set $\left\{x \in \mathbb{R}^{n}: \phi(x) \leq \frac{1}{2 \tau}\right\} \equiv B\left(\tau, \sigma_{0}\right)$ where $\tau$ is uniquely determined by the test condition

$$
\int \chi_{B\left(\tau, \sigma_{0}\right)}(x) d \mu(x)=\sigma_{0}^{2} u+v .
$$

Since the latter integral is an increasing function of $\tau$ and since $\tau_{0}$ satisfies (60), we conclude that $\rho_{0}=\chi_{B\left(\tau, \sigma_{0}\right)}$ is a maximizer in (59). This yields

$$
A=\int \rho_{0}(x) \phi(x) d \mu(x)=\int \frac{x_{1} \rho_{0}}{|x|^{n+2-\alpha}} d_{\omega} x .
$$

Combining the obtained inequalities and using (58) implies

$$
\mathscr{N}_{\alpha}^{+}(u, v)=\sigma_{0}^{2(\alpha-1)} h_{\alpha}^{2}\left(t_{0}\right) \equiv u^{2(\alpha-1) / \alpha} \frac{h_{\alpha}^{2}\left(t_{0}\right)}{f_{\alpha}^{2(\alpha-1) / \alpha}\left(t_{0}\right)}
$$

Step 2 . We claim that $\mathscr{N}_{\alpha}^{+}(u, v)$ defined implicitly by (61) is an increasing function of each argument separately. It suffices to verify this for an auxiliary function $G(u, v)=\left(\mathscr{N}_{\alpha}^{+}(u, v)\right)^{\alpha / 2}$. On eliminating $\sigma_{0}$ by virtue of (56) we obtain

$$
G(u, v)=u^{\alpha-1} g_{1}\left(t_{0}\right)=v^{\frac{\alpha(1-\alpha)}{2-\alpha}} g_{2}\left(t_{0}\right)^{\frac{\alpha}{2-\alpha}}
$$

where

$$
g_{1}(t)=\frac{h_{\alpha}^{\alpha}(t)}{f_{\alpha}^{\alpha-1}(t)}, \quad g_{2}(t)=\frac{h_{\alpha}^{2-\alpha}(t)}{f_{\alpha-2}^{1-\alpha}(t)}
$$

Using (31) and (32) yields for the logarithmic derivatives

$$
\begin{aligned}
\frac{g_{1}^{\prime}}{g_{1}} & =\frac{\alpha h_{\alpha}^{\prime} f_{\alpha}-(\alpha-1) f_{\alpha}^{\prime} h_{\alpha}}{f_{\alpha} h_{\alpha}}=\frac{\alpha f_{\alpha-2}^{\prime} f_{\alpha}-(\alpha-2) f_{\alpha}^{\prime} f_{\alpha-2}}{2 t f_{\alpha} h_{\alpha}} \\
& =\frac{g^{\prime}}{g} \cdot \frac{f_{\alpha-2}}{2 t h_{\alpha}}>0 \\
\frac{g_{2}^{\prime}}{g_{2}} & =\frac{(2-\alpha) h_{\alpha}^{\prime} f_{\alpha-2}+(\alpha-1) f_{\alpha-2}^{\prime} h_{\alpha}}{f_{\alpha-2} h_{\alpha}}=\frac{\alpha f_{\alpha} h_{\alpha}^{\prime}-(\alpha-1) f_{\alpha}^{\prime} h_{\alpha}}{f_{\alpha-2} h_{\alpha}} \\
& =\frac{g_{1}^{\prime}}{g_{1}} \cdot \frac{f_{\alpha}}{f_{\alpha-2}}>0
\end{aligned}
$$

which implies that $g_{1}$ and $g_{2}$ are increasing functions of $t$ for any $0<\alpha \leq 2$. Now suppose that $u_{1} \geq u$ and $v_{1} \geq v$. Let $t_{1}$ be the unique solution of $g\left(t_{1}\right)=u_{1}^{\alpha-2} v_{1}^{\alpha}$. Since $g(t)$ in (57) is increasing we conclude that $t_{1} \geq t$. First let us consider $1 \leq \alpha \leq 2$. Then using the first equality in (62) yields 


$$
G(u, v)=u^{\alpha-1} g_{1}\left(t_{0}\right) \leq u_{1}^{\alpha-1} g_{1}\left(t_{1}\right)=G\left(u_{1}, v_{1}\right)
$$

Similarly, if $0<\alpha \leq 1$ then using the second equality in (62) yields

$$
G(u, v)=v^{\frac{\alpha(1-\alpha)}{2-\alpha}} g_{2}\left(t_{0}\right)^{\frac{\alpha}{2-\alpha}} \leq v_{1}^{\frac{\alpha(1-\alpha)}{2-\alpha}} g_{2}\left(t_{1}\right)^{\frac{\alpha}{2-\alpha}}=G\left(u_{1}, v_{1}\right) \text {. }
$$

The claim follows.

Step 3. Now suppose that $\rho$ is an arbitrary density with a compact support not containing the origin. We claim that $\mathscr{N}_{\alpha}^{+}(u, v) \leq \mathscr{N}_{\alpha}(u, v)$. Let $U_{ \pm}$and $U_{0}$ denote the classes of densities satisfying respectively the conditions

(a) $\operatorname{supp} \rho \cap \mathbb{R}_{\mp x_{1}>0}^{n}$ has measure zero;

(b) both supp $\rho \cap \mathbb{R}_{x_{1}>0}^{n}$ and supp $\rho \cap \mathbb{R}_{x_{1}<0}^{n}$ have nonzero measures.

Let $\mathscr{N}_{\alpha}^{*}(u, v)$ denote the supremum in (16) taken over the corresponding class of densities. Then

$$
\mathscr{N}_{\alpha}(u, v)=\max \left\{\mathscr{N}_{\alpha}^{-}(u, v), \mathscr{N}_{\alpha}^{+}(u, v), \mathscr{N}_{\alpha}^{0}(u, v)\right\},
$$

Let $x \rightarrow \tilde{x}$ be the reflection about the hyperplane $x_{1}=0$. Then $\widetilde{\rho}(x):=\rho(\tilde{x})$ is a bijection between $U_{+}$and $U_{-}$. This implies $\mathscr{N}_{\alpha}^{-}(u, v)=\mathscr{N}_{\alpha}^{+}(u, v)$. Let $\rho \in U^{0}$ and let $\rho^{ \pm}(x)=\chi_{\mathbb{R}_{ \pm x_{1}>0}^{n}} \rho(x)$ such that $\rho^{ \pm}(x) \in U^{ \pm}$and $\rho=\rho^{+}+\rho^{-}$a.e. in $\mathbb{R}^{n}$. We have

$$
\begin{aligned}
\left|\mathcal{H}_{\alpha}(\rho)\right|^{2} & =\left|\mathcal{H}_{\alpha}\left(\rho^{+}\right)-\mathcal{H}_{\alpha}\left(\widetilde{\rho^{-}}\right)\right|^{2} \leq \max \left\{\mathcal{H}_{\alpha}\left(\rho^{+}\right)^{2}, \mathcal{H}_{\alpha}\left(\widetilde{\rho^{-}}\right)^{2}\right\} \\
& \leq \mathcal{H}_{\alpha}\left(\rho_{1}\right)^{2} \leq \mathcal{N}_{\alpha}^{+}\left(u_{1}, v_{1}\right),
\end{aligned}
$$

where $\rho_{1}$ is one of $\rho^{+}$and $\widetilde{\rho^{-}}$, and $\mathcal{F}_{\alpha} \rho_{1}=u_{1} \leq u, \mathcal{F}_{\alpha-2} \rho_{1}=v_{1} \leq v$. By the monotonicity of $\mathscr{N}_{\alpha}^{+}$,

$$
\left|\mathcal{H}_{\alpha}(\rho)\right|^{2} \leq \mathscr{N}_{\alpha}^{+}(u, v)
$$

implying $\mathscr{N}_{\alpha}^{0}(u, v) \leq \mathscr{N}_{\alpha}^{+}(u, v)$. This proves our claim and, thus, finishes the proof of the theorem.

\section{Some applications}

First we demonstrate how Theorem 2 implies Theorem 1 . To this end consider $\alpha=2$.

\section{Corollary 2}

$$
\mathscr{N}_{2}(u, v)=u \mathscr{M}_{n}(v)
$$

where $\mathscr{M}_{n}(t)$ is the unique solution of the initial problem (3). 
Proof By (46),

$$
f_{2}(t)=t^{2-n}\left(t^{2}-1\right)^{n / 2} F\left(0,2 ; \frac{1}{2} n+1,1-t^{2}\right)=t^{2-n}\left(t^{2}-1\right)^{n / 2}
$$

and by $(31) h_{2}(t)=f_{2}(t) / t$, therefore Theorem 2 yields

$$
\mathscr{N}_{\alpha}(u, v)=u \frac{h_{2}^{2}(t)}{f_{2}(t)}=u\left(1-\frac{1}{t^{2}}\right)^{n / 2},
$$

where $t=t(v)$ is uniquely determined by virtue of $f_{0}(t)=v$. Define

$$
\mathscr{M}(v)=\left(1-t(v)^{-2}\right)^{n / 2}
$$

such that

$$
\mathscr{N}_{\alpha}(u, v)=u \mathscr{M}_{n}(v)
$$

To establish (3), we notice that $\mathscr{M}_{n}(0)=0$ and also, using (32), one has from (64)

$$
f_{0}^{\prime}(t)=2 t h_{2}^{\prime}(t)-f_{2}^{\prime}(t)=f_{2}\left(\frac{2 h_{2}^{\prime}}{h_{2}}-\frac{f_{2}^{\prime}}{f_{2}}\right)=n t^{1-n}\left(t^{2}-1\right)^{(n-2) / 2},
$$

therefore,

$$
\frac{d \mathscr{M}_{n}}{d v}=\frac{d \mathscr{M}_{n}}{d t} \cdot \frac{1}{f_{0}^{\prime}(t)}=\frac{1}{t^{2}}=1-\mathscr{M}_{n}^{2 / n}
$$

as desired.

\section{Proof of Theorem 3}

First we assume that dimension $n \geq 2$ is chosen arbitrarily. Since $\alpha=1$, we have by the inversion invariance (31) that $f_{-1}(t)=f_{1}(t)$, which eliminates function $f_{-1}(t)$ from the consideration. Then (32) and (33) amount to

$$
\begin{aligned}
t h_{1}^{\prime}(t) & =f_{1}^{\prime}(t), \\
(n-1) h_{1}(t) & =\left(t^{2}-1\right) f_{1}^{\prime}-t f_{1}(t),
\end{aligned}
$$

where by virtue of the hypergeometric representations (46) and (50) we have respectively

$$
\begin{gathered}
f_{1}(t)=t^{2-n}\left(t^{2}-1\right)^{n / 2} F\left(\frac{1}{2}, \frac{3}{2} ; \frac{1}{2} n+1,1-t^{2}\right) \\
h_{1}(t)=t^{1-n}\left(t^{2}-1\right)^{n / 2} F\left(\frac{1}{2}, \frac{1}{2} ; \frac{n+2}{2}, 1-t^{2}\right)
\end{gathered}
$$


Furthermore, by the definition $f_{1}(1)=h_{1}(1)=0$. Also, since $n \geq 2$, one finds by l'Hospital's rule from (37) that

$$
h_{1}^{\prime}(1)=f_{1}^{\prime}(1)=n \cdot \lim _{t \rightarrow 1+0} t^{2-n}\left(t^{2}-1\right)^{(n-2) / 2}=\left\{\begin{array}{l}
2, n=2 \\
0, n \geq 3 .
\end{array}\right.
$$

Applying Theorem 2 we obtain

Lemma 3 If $u, v>0$ then the supremum

$$
\mathscr{N}_{1}(u, v):=\sup \left\{\left|\mathcal{H}_{1} \rho\right|^{2}: \mathcal{F}_{1} \rho=u, \quad \mathcal{F}_{-1} \rho=v, \quad 0 \leq \rho \leq 1\right\}
$$

is given by formula

$$
\mathscr{N}_{1}(u, v)=\Phi_{n}(u v)
$$

where $\Phi_{n}(s)$ is well-defined by

$$
\left\{\begin{array}{l}
s=t^{2-n}\left(t^{2}-1\right)^{n / 2} F\left(\frac{1}{2}, \frac{3}{2} ; \frac{1}{2} n+1,1-t^{2}\right) \\
\Phi_{n}(s)=t^{-n-1}\left(t^{2}-1\right)^{n / 2} F\left(\frac{1}{2}(n+1), \frac{3}{2} ; \frac{1}{2} n+1, \frac{t^{2}-1}{t^{2}}\right)
\end{array}\right.
$$

Alternatively, $\Phi_{n}(s)$ is the unique solution of the singular initial problem (24)-(25).

Proof Since $f_{-1}=f_{1}$, it follows from Theorem 2 that $\mathscr{N}_{1}(u, v)=h_{1}(t)$, where $1<t<\infty$ is (uniquely) determined by $f_{1}(t)=\sqrt{u v}$, hence

$$
\mathscr{N}_{1}(u, v)=\left(h_{1} \circ f_{1}^{-1}\right)(\sqrt{u v}) .
$$

Let us consider the composed function $\Phi_{n}(s)=\left(h_{1} \circ f_{1}^{-1}\right)(s)$. Obviously, $\Phi_{n}(s)$ is an increasing function. By the definition, $\Phi_{n}$ is determined by the parametric representation

$$
\left\{\begin{array}{l}
s=f_{1}(t) \\
\Phi(s)=h_{1}(t)
\end{array}\right.
$$

therefore $\Phi_{n}(0)=0$ and by (45),

$$
\lim _{z \rightarrow 1} \frac{f_{1}(t)}{t}=c_{n}:=\frac{\Gamma\left(\frac{n+2}{2}\right)}{\Gamma\left(\frac{n+1}{2}\right) \Gamma\left(\frac{3}{2}\right)} \equiv \frac{\omega_{1} \omega_{n-1}}{\omega_{n}} .
$$

Then it follows by l'Hospital's rule from $(65)_{1}$ that

$$
\lim _{s \rightarrow \infty} \frac{\Phi_{n}(s)}{\ln s}=\lim _{t \rightarrow \infty} \frac{h_{1}(t)}{\ln f_{1}(t)}=\lim _{t \rightarrow \infty} \frac{h_{1}^{\prime}(t) f_{1}(t)}{f_{1}^{\prime}(t)}=\lim _{t \rightarrow \infty} \frac{f_{1}(t)}{t}=c_{n},
$$

hence

$$
\Phi_{n}(s) \sim c_{n} \ln s \quad \text { as } s \rightarrow \infty
$$


Further, we find from $(65)_{1}$ that $\Phi_{n}^{\prime}(s)=h_{1}^{\prime}(t) / f_{1}^{\prime}(t)=1 / t$, hence $\Phi_{n}^{\prime}(0)=1$ and applying the chain rule and $(65)_{2}$ yields

$$
\Phi_{n}^{\prime \prime}(s)=-\frac{1}{t^{2}} \frac{d t}{d s}=-\frac{1}{t^{2}} \frac{1}{f_{1}^{\prime}(t)}=\frac{\frac{1}{t^{2}}-1}{\left.(n-1) h_{1}(t)+t f_{1}(t)\right)}=\frac{\Phi_{n}^{\prime}\left(\Phi_{n}^{\prime 2}-1\right)}{(n-1) \Phi_{n} \Phi_{n}^{\prime}+s} .
$$

This shows that $\Phi_{n}$ verifies the conditions (24)-(25). It follows from the above pearametric representation that a solution of the singular problem (24)-(25) is unique. The lemma follows.

We finish this section by some comments on the particular cases $n=1$ and $n=2$. If $n=1$ then analysis here is straightforward. Using (51), Theorem 2 implies the following new inequality, see (13) and the discussion afterwards.

Corollary 3 For any measurable function $0 \leq \rho(x) \leq 1, x \in \mathbb{R}$, and $0 \notin \operatorname{supp} \rho$, there holds

$$
\sinh ^{2}\left(\int_{-\infty}^{\infty} \frac{\rho(x)}{x} d x\right) \leq \int_{-\infty}^{\infty} \rho(x) d x \int_{-\infty}^{\infty} \frac{\rho(x)}{x^{2}} d x .
$$

The inequality is sharp and attained whenever $\rho(x)$ is a characteristic function of an interval $[a, b]$ with $a b>0$.

Proof It follows from (51) that $f_{1}(\xi)=\sinh \xi$ and $h_{1}(t)=\xi$, where $t=\cosh \xi$. In particular,

$$
\int_{-\infty}^{\infty} \frac{\rho(x)}{x} d x \leq \ln \left(\xi+\sqrt{1+\xi^{2}}\right)
$$

where

$$
\xi^{2}=\int_{-\infty}^{\infty} \rho(x) d x \int_{-\infty}^{\infty} \frac{\rho(x)}{x^{2}} d x
$$

Coming back to the Markov type inequality (14) involving $s_{-1}$, we obtain a further extension: in the notation of (9) we have

$$
\sinh ^{2} s_{-1} \leq s_{0} s_{-2}
$$

Next, note that the case $n=2$ is special in several respects. First, the derivative $f_{1}^{\prime}(1)=2$ is nonzero by virtue of (68). Furthermore, when $n=2$, the function $\Phi_{2}$ defined by (24) has some extra symmetries due to $n-1=1$. In this case, the defining functions $f_{1}$ and $h_{1}$ admit a nice parameterizations by virtue of complete elliptic integrals. We confine ourselves to the following remarkable Taylor expansion at the origin of $\Phi_{2}(z)$ :

$$
\begin{aligned}
\Phi_{2}(z)= & z-\frac{1}{2^{2}} z^{2}+\frac{1}{2^{4}} z^{3}-\frac{7}{2^{9}} z^{4}+\frac{5}{2^{11}} z^{5}-\frac{21}{2^{16}} z^{6} \\
& +\frac{3}{2^{17}} z^{7}+\frac{7}{2^{24}} z^{8}+\frac{11}{2^{26}} z^{9}-\frac{959}{2^{32}} z^{10}+\ldots
\end{aligned}
$$


Acknowledgements The author thank the referee for the valuable comments.

\section{Compliance with ethical standards}

Conflict of interest The author has no conflict of interest.

Open Access This article is distributed under the terms of the Creative Commons Attribution 4.0 International License (http://creativecommons.org/licenses/by/4.0/), which permits unrestricted use, distribution, and reproduction in any medium, provided you give appropriate credit to the original author(s) and the source, provide a link to the Creative Commons license, and indicate if changes were made.

\section{References}

1. Abramowitz, M., Stegun, I.A.: Handbook of mathematical functions with formulas, graphs, and mathematical tables. National Bureau of Standards Applied Mathematics Series, vol. 55. For Sale by the Superintendent of Documents, U.S. Government Printing Office, Washington, D.C. (1964). http:// people.math.sfu.ca/ cbm/aands/frameindex.htm

2. Adams, D.R.: A note on Riesz potentials. Duke Math. J. 42(4), 765-778 (1975). http://projecteuclid. org/euclid.dmj/1077311348

3. Adams, D.R., Hedberg, L.I.: Function Spaces and Potential Theory, Grundlehren der Mathematischen Wissenschaften [Fundamental Principles of Mathematical Sciences]. Springer, Berlin (1996). https:// doi.org/10.1007/978-3-662-03282-4

4. Aheizer, N.I., Krein, M.: Some questions in the theory of moments. Translated by W. Fleming and D. Prill. Translations of Mathematical Monographs, Vol. 2. American Mathematical Society, Providence (1962)

5. Curto, R.E., Fialkow, L.A.: Truncated $K$-moment problems in several variables. J. Oper. Theory 54(1), 189-226 (2005)

6. Fraenkel, L.E.: An Introduction to Maximum Principles and Symmetry in Elliptic Problems, Cambridge Tracts in Mathematics, vol. 128. Cambridge University Press, Cambridge (2000). https://doi.org/10. 1017/CBO9780511569203

7. Garg, R., Spector, D.: On the role of Riesz potentials in Poisson's equation and Sobolev embeddings. Indiana Univ. Math. J. 64(6), 1697-1719 (2015). https://doi.org/10.1512/iumj.2015.64.5706

8. Gustafsson, B., He, C., Milanfar, P., Putinar, M.: Reconstructing planar domains from their moments. Inverse Prob. 16(4), 1053-1070 (2000). https://doi.org/10.1088/0266-5611/16/4/312

9. Gustafsson, B., Putinar, M.: The exponential transform: a renormalized Riesz potential at critical exponent. Indiana Univ. Math. J. 52(3), 527-568 (2003). https://doi.org/10.1512/iumj.2003.52.2304

10. Gustafsson, B., Putinar, M.: Hyponormal quantization of planar domains: complex orthogonal polynomials and the exponential transform. Lecture Notes in Mathematics, vol. 2199. Springer (2017)

11. Gustafsson, B., Putinar, M., Saff, E.B., Stylianopoulos, N.: Bergman polynomials on an archipelago: estimates, zeros and shape reconstruction. Adv. Math. 222(4), 1405-1460 (2009). https://doi.org/10. 1016/j.aim.2009.06.010

12. Gustafsson, B., Vasil'ev, A.: Conformal and Potential Analysis in Hele-Shaw Cells. Advances in Mathematical Fluid Mechanics. Birkhäuser Verlag, Basel (2006)

13. Huang, X.: Symmetry results of positive solutions of integral equations involving Riesz potential in exterior domains and in annular domains. J. Math. Anal. Appl. 427(2), 856-872 (2015). https://doi. org/10.1016/j.jmaa.2015.02.083

14. Huang, X., Hong, G., Li, D.: Some symmetry results for integral equations involving Wolff potential on bounded domains. Nonlinear Anal. 75(14), 5601-5611 (2012). https://doi.org/10.1016/j.na.2012. 05.007

15. Krein, M.G., Nudel'man, A.A.: The Markov moment problem and extremal problems. American Mathematical Society, Providence, R.I. (1977). Ideas and problems of P. L. Čebyšev and A. A. Markov and their further development, Translated from the Russian by D. Louvish, Translations of Mathematical Monographs, vol. 50 (1977)

16. Lasserre, J.B.: Bounding the support of a measure from its marginal moments. Proc. Am. Math. Soc. 139(9), 3375-3382 (2011). https://doi.org/10.1090/S0002-9939-2011-10865-7 
17. Lieb, E.H., Loss, M.: Analysis, Graduate Studies in Mathematics, vol. 14, 2nd edn. American Mathematical Society, Providence (2001). https://doi.org/10.1090/gsm/014

18. Maz'ya, V.: Sobolev spaces with applications to elliptic partial differential equations, Grundlehren der Mathematischen Wissenschaften [Fundamental Principles of Mathematical Sciences], vol. 342, augmented edn. Springer, Heidelberg (2011). https://doi.org/10.1007/978-3-642-15564-2

19. Maz'ya, V., Shaposhnikova, T.: On pointwise interpolation inequalities for derivatives. Math. Bohem. 124(2-3), 131-148 (1999)

20. Mingione, G.: Gradient potential estimates. J. Eur. Math. Soc. (JEMS) 13(2), 459-486 (2011). https:// doi.org/10.4171/JEMS/258

21. Mingione, G.: Recent Advances in Nonlinear Potential Theory Trends in Contemporary Mathematics. Springer INdAM Ser., vol. 8, pp. 277-292. Springer, Cham (2014)

22. Reichel, W.: Characterization of balls by Riesz-potentials. Ann. Mat. Pura Appl. (4) 188(2), 235-245 (2009). https://doi.org/10.1007/s10231-008-0073-6

23. Tkachev, V.G.: Subharmonicity of higher dimensional exponential transforms. In: Ebenfelt, P., Gustafsson, B., Khavinson, D., Putinar, M. (eds) Quadrature Domains and Their Applications, Oper. Theory Adv. Appl., vol. 156, Birkhäuser, Basel (2005)

24. Xiao, J.: Entropy flux-electrostatic capacity-graphical mass. Proc. Am. Math. Soc. 145(2), 825-832 (2017). https://doi.org/10.1090/proc/13259 